\title{
A ocorrência de dor musculoesquelética em professores da rede pública estadual da Bahia.
}

The occurrence of musculoskeletal pain in state public school teachers in Bahia

\author{
Claudiney André Leite Pereira ${ }^{1 *}$
}

\begin{abstract}
RESUMO
Tivemos como objetivo avaliar a presença de dores musculoesquelética em docentes da rede estadual da Bahia e suas possíveis relações com os níveis de flexibilidade. Tratou-se de uma pesquisa descritiva onde participaram do estudo 25 docentes da Rede Estadual da Bahia que atuam na mesorregião metropolitana de Salvador. Realizou-se a coleta de dados através do questionário do Diagrama das áreas dolorosas para obter informações sobre intensidades das dores musculares e o teste do banco de wells para medir o nível de flexibilidade. Como tratamento estatístico utilizou-se o coeficiente de Pearson para verificar o nível de correlação das variáveis. Observou-se que $52 \%$ dos professores apresentam um baixo nível de flexibilidade, mas do ponto de vista estatístico não podemos afirmar neste estudo que existe uma relação consistente entre a dor muscular e os níveis de flexibilidade. Identificamos há necessidade de mais estudos para se ter um melhor diagnostico sobre este problema.
\end{abstract}

Palavras - Chave: Dor; Flexibilidade; Professores.

\begin{abstract}
We aimed to evaluate the presence of musculoskeletal pain in teachers from the state network of Bahia and its possible relationship with the levels of flexibility. It was a descriptive research in which 25 teachers from the State Network of Bahia who work in the metropolitan mesoregion of Salvador participated in the study. Data were collected through the Painful Area Diagram questionnaire to obtain information on muscle pain intensities and the wells bank test to measure the level of flexibility. As statistical treatment, the Pearson coefficient was used to verify the level of correlation of the variables. It was observed that $52 \%$ of teachers have a low level of flexibility, but from a statistical point of view, we cannot affirm in this study that there is a consistent relationship between muscle pain and flexibility levels. We identified the need for more studies to have a better diagnosis of this problem.
\end{abstract}

Keywords: Pain; Flexibility; Teachers.

\footnotetext{
${ }^{1}$ Instituto Federal Baiano 1. *E-mail: eu-ney@ hotmail.com
} 


\section{INTRODUÇÃO}

Existem muitos estudos que se dedicam a investigar os problemas que envolvem as atividades dos docentes principalmente os relacionadas as dores musculares, considerando também que existem outras questões que comprometem a saúde destes trabalhadores como: as emocionais, sociais, psicossomáticas e posturais. (SOUZA et al., 2017).

Em termos de qualidade de vida no trabalho, envolvem questões que vão além do ambiente laboral, mas também a vida do trabalhador em todo o seu ciclo social. (PEREIRA, 2020)

Geralmente os professores trabalham em regime de 40hs semanais, em muitos casos em escolas com salas apertadas e cheias de alunos, pouco material de apoio levando os mesmos a terem que escrever no quadro as atividades dos discentes, além disso, exercem atividades repetitivas em casa como correção de provas ou longas horas de pesquisas no computador (TACON et al.,2016)

Além dos aspectos que envolvem as estruturas físicas do ambiente laboral dos professores, alguns trabalham em área de risco social onde a carência socioeconômica dos alunos e seus possíveis envolvimentos com drogas ou violência doméstica acaba tendo impacto na sala de aula o que pode ter como consequência o desencadeamento de diversas doenças de ordem psicossomática nos docentes (COSTA et al.,2014)

Além destes problemas elencados, existem os relacionados as dores músculo esqueléticas que são bastantes comuns em trabalhadores que passam muito tempo em uma mesma posição como os professores que exercem suas atividades laborais em posições geralmente com pouca movimentação o que acabam gerando tensões e incômodos (BRASIL, 2012).

Os professores durante a sua atividade profissional passam muito tempo com o membro superior abduzidos o que acaba gerando desconforto, os membros inferiores também sofrem com posições estáticas tendendo a rigidez, pessoas que trabalham muito tempo em uma posição, seja sentado ou em pé, tendem a sofrer dores principalmente pelo encurtamento dos isquiotibiais (POLACHINI et al.,2005) 
Quando se trata de docentes geralmente estas dores aparecem na região do tronco, principalmente por ser uma região bastante exigidas nas atividades de leitura e escrita, a dor nesta região parece ser uma característica bastante presente nestes profissionais (BRANCO et al., 2011).

O trabalho com movimentos repetitivos muitas vezes feitos em posições incorretas pode levar as dores musculares principalmente se este profissional não tem o hábito de uma atividade física regular, que poderia funcionar como forma de compensação para possíveis sobrecargas unilaterais.

A dificuldade de reservar um tempo para a pratica de uma atividade física regular é um problema que envolve o trabalhador no mundo moderno, o que os deixa vulneráveis a problemas de saúde, alguns trabalhadores demonstram mais propensão ao aparecimento de dores na estrutura musculoesquelética relacionada ao trabalho. (BRASIL 2012).

Muitos professores além da manutenção de posturas repetitivas nas atividades laborais, sofrem também com o sedentarismo e as vezes em casa ficam muito tempo em aparelhos eletrônicos com atividades de pesquisa o que termina contribuindo para o aparecimento de dores, principalmente por uma falta de atividade física nas horas de lazer (BORSOI, 2012).

Considerando as atividades profissionais dos professores não terminam na sala de aula, muitos acabam adquirindo um ritmo de vida com pouco movimento o que pode levar ao aparecimento de dores principalmente as lombares, sabemos que os exercícios físicos podem se tornar um importante elemento na prevenção dessa patologia (SANTNDAERT et al., 2011).

A Organização Mundial da Saúde (OMS) estabelece que para os adultos a prática de atividade física seja no mínimo 150 minutos por semana de atividade física moderada ou 75 minutos por semana de esforço mais vigoroso, em períodos de pelo menos 10 minutos, sem considerar da frequência semanal (BULL et al., 2020).

Estes parâmetros estabelecidos pela OMS procuram garantir níveis satisfatórios das aptidões físicas relacionadas a saúde, que segundo GUEDES e GUEDES (1995) essas aptidões correspondem as dimensões morfológicas, funcional-motora, fisiológica e comportamental, sendo que a flexibilidade se encontra na dimensão funcional motora. 
O baixo nível de flexibilidade pode estar relacionado ao aparecimento de algumas dores já que a falta de uma boa amplitude articular pode comprometer a mecânica dos movimentos e a postura corporal, levando as pessoas a adoção de posições incorretas. A flexibilidade é uma valência física que estar presente tanto nas atividades físicas quanto nas atividades da vida diária. Para MONTEIRO e FARINATTI (1999) ter um mínimo dessa valência física possibilita uma maior mobilidade e diminuição dos riscos de lesões.

Manter uma boa funcionalidade em todas as articulações, garante a eficácia dos movimentos corporais; neste caso a flexibilidade torna-se um componente importante do condicionamento físico, considerando o corpo como uma cadeia cinética, qualquer rigidez nas articulações pode limitar a amplitude de movimento afetando a coluna vertebral. (HOWLEY; FRANKS, 2008).

Diante deste quadro o objetivo deste trabalho foi avaliar a presença de dor em docentes da rede estadual que atuam na mesorregião metropolitana de Salvador -Ba e suas possíveis relações com os níveis de flexibilidade.

\section{MATERIAL E MÉTODOS}

O estudo tratou-se de uma pesquisa descritiva que segundo (GIL, 2008) tem por objetivo identificar o perfil e as características de um grupo e as relações entre as variáveis e a natureza destes eventos que serão investigados na população em estudo.

Foram incluídos na pesquisa profissionais da educação que trabalham na rede estadual de ensino que atuam na mesorregião metropolitana de Salvador, selecionamos uma escola, apresentamos o trabalho ao corpo docente e marcamos a data das avaliações.

Participaram do trabalho 25 docentes, as etapas da pesquisa consistiram em primeiro os professores responderam ao questionário com os dados pessoais, em seguida preencheram o diagrama das áreas dolorosas e por fim realizaram o teste de flexibilidade.

Através do questionário foram investigadas questões sobre tempo de docência, sexo, idade, regime de trabalho, tempo de serviço e nível de flexibilidade, através destas informações foi estabelecido o perfil dos entrevistados.

As entrevistas foram feitas ao longo de quatro dias no período matutino durante o intervalo das aulas para que não comprometessem as atividades da escola e as aulas dos discentes. 
Para registro das dores, usamos o diagrama das áreas dolorosas de (CORLETT; MANENICA, 1980) onde o corpo humano estava dividido em 21 regiões nas quais os professores sinalizavam se sentiam dor e qual a sua intensidade, conforme a Tabela.1, este questionário é usado para identificar as regiões e os níveis de intensidade de dor nas articulações do corpo humano (LIDA, 2012).

Tabela 1. Níveis de intensidade de dor.

\begin{tabular}{|l|l|l|l|l|}
\hline \multicolumn{5}{|c|}{ Intensidade } \\
\hline 1 & 2 & 3 & 4 & 5 \\
\hline Nenhum & Algum & Moderado & Bastante & Intolerável \\
Desconforto/dor & Desconforto/dor & Desconforto/dor & Desconforto/dor & Desconforto/dor \\
\hline
\end{tabular}

Fonte: CORLETT; MANENICA, 1980.

Existem vários métodos que servem para avaliar os níveis de flexibilidade, utilizamos o teste do banco de Wells conhecido também como, teste de sentar e alcançar, que é um método proposto por WELLS e DILLON, (1952), por ser de seguro, de fácil aplicabilidade e apresentar resultados confiáveis.

Este teste consiste em o indivíduo sentando no chão em frente ao banco com as pernas estendidas, onde sem flexionar os joelhos através do movimento da flexão do tronco o avaliado vai tocar em uma escala registrada em centímetros na parte superior do banco na maior distância possível, foram feitas três tentativas sendo considerada a de valor mais alto, seu desempenho foi avaliado de acordo com a Tabela 2

Tabela 2. Tabela do Teste de Flexibilidade

\begin{tabular}{|l|l|l|l|l|l|l|}
\hline \multicolumn{7}{|l|}{ Sentar e Alcançar - Masculino - com banco (em Centímetros) } \\
\hline Idade & $15-19$ & $20-29$ & $30-39$ & $40-49$ & $50-59$ & $60-69$ \\
\hline Excelente & $>39$ & $>40$ & $>38$ & $>35$ & $>35$ & $>33$ \\
\hline Acima da média & $34-38$ & $34-39$ & $33-37$ & $29-34$ & $28-34$ & $25-32$ \\
\hline Média & $29-33$ & $30-33$ & $28-32$ & $24-28$ & $24-27$ & $20-24$ \\
\hline Regular & $24-28$ & $25-29$ & $23-27$ & $18-23$ & $16-23$ & $15-19$ \\
\hline Fraco & $<23$ & $<24$ & $<22$ & $<17$ & $<15$ & $<14$ \\
\hline Sentar e Alcançar - Feminino-com banco (em Centímetros) \\
\hline Idade & $15-19$ & $20-29$ & $30-39$ & $40-49$ & $50-59$ & $60-69$ \\
\hline Excelente & $>43$ & $>41$ & $>41$ & $>38$ & $>39$ & $>35$ \\
\hline Acima da média & $38-42$ & $37-40$ & $36-40$ & $34-37$ & $33-38$ & $31-34$ \\
\hline Média & $34-37$ & $33-36$ & $32-35$ & $30-33$ & $30-32$ & $27-30$ \\
\hline Regular & $29-33$ & $28-32$ & $27-31$ & $25-29$ & $25-29$ & $23-26$ \\
\hline Fraco & $<28$ & $<27$ & $<26$ & $<24$ & $<24$ & $<22$ \\
\hline
\end{tabular}

Fonte: WELLS; DILLON, 1952. 
Como tratamento estatístico usamos o coeficiente de Pearson, onde os valores de (r) encontrados podem variar de -1 a 1 , sendo $\mathbf{r}=1$ Significa uma correlação perfeita positiva entre as duas variáveis e $\mathbf{r}=-1$ Significa uma correlação negativa perfeita entre as duas variáveis, isto é, se uma aumenta, a outra sempre diminui. Utilizamos os recursos estatísticos da planilha Excel para realização dos cálculos e gráficos. Fizemos a correlação da dor com a flexibilidade e a dor associada com a idade.

\section{RESULTADOS}

Procuramos descrever através do preenchimento de um questionário o perfil dos participantes, conforme Tabela 3 entrevistamos 25 docentes divididos entre $24 \%$ de homens e $76 \%$ de mulheres, é uma característica muito presente na educação a maior parte do professorado sem composta pelo sexo feminino. (CARVALHO ,2018).

Esta característica se acentua ainda mais em escolas no interior do Brasil, onde geralmente os homens saem de casa mais cedo para trabalhar geralmente em centros maiores com mais possibilidades de emprego e as mulheres terminam se dedicando mais aos estudos e fazendo o magistério que é um curso de nível médio ainda muito presente nas escolas públicas.

Em relação ao tempo de serviço $80 \%$ tem acima de 15 anos de atividade docente, sendo a faixa etária mais presente foi de 40 anos a 49 anos que corresponde a $56 \%$ dos entrevistados. A idade e o tempo de trabalho na escola, estão muitos associados a dor muscular esquelética. (JEFFERSON, 2009).

Geralmente os professores da rede pública exercem sua profissão até a aposentadoria, o que os leva a ter um longo tempo de trabalho docente predispondo-os a doenças musculoesqueléticas, podendo estar associadas a dores de alta intensidade. (JEFFERSON, 2009). 
Tabela.3 Perfil dos Entrevistados

\begin{tabular}{lcr}
\hline Variável & N & $\%$ \\
\hline Homens & 6 & 24 \\
Mulheres & 18 & 76 \\
Tempo de Serviço & & \\
5 anos a 10 anos & 2 & 8 \\
10 anos a 15 anos & 3 & 12 \\
acima de 15 anos & 20 & 80 \\
Faixa Etária & & \\
30 anos a 39 anos & 6 & 24 \\
40 anos a 49 anos & 13 & 56 \\
acima de 50 anos & 6 & 24 \\
RegimeTrabalho & & \\
& & \\
40 hs. & 25 & 52 \\
Nível de Flexibilidade & & 28 \\
Fraco & 12 & 20 \\
Regular & 7 & \\
Médio & 6 & \\
DOR & 10 & \\
Nenhuma Dor a Dor Moderada & & \\
Alguma Dor a Dor intolerável & 15 & \\
\hline
\end{tabular}

Fonte: PEREIRA, 2021.

Todos os professores trabalham em regime de 40hs, exercendo uma carga horaria grande nesta escola, devemos ressaltar que a atividade docente não termina na escola quando consideramos a necessidade de preparação das aulas, provas, conteúdos etc. (FERNANDES et al., 2009).

Geralmente as atividades docentes envolvem poucos movimentos sendo então realizadas de forma sedentária o que leva a estes profissionais a predisposição a doenças hipocinéticas dentre elas as dores musculares, a falta da prática de atividade física regular termina levando a diminuição da amplitude articular ocasionando tensão muscular.

Quando o indivíduo não aproveita a totalidade de sua amplitude de movimento articular, o tecido tendíneo para compensar sofre encurtamento, o que termina comprometendo os movimentos necessários as atividades da vida diária, se posturas inadequadas forem 
mantidas em excesso, o músculo pode sofrer um encurtamento gerando lesões na coluna quando forem solicitados em sua amplitude de movimento. (HOWLEY; FRANKS, 2008).

Ao avaliarmos o nível de flexibilidade, verificamos que $52 \%$ foram classificados como fracos nesta valência Física, 28\% regular e apenas 20\% médio considerando que classificação máxima neste teste é excelente, ter um bom nível de mobilidade e amplitude articular garante uma boa mecânica dos movimentos, encurtamentos e enrijecimentos musculares terminam gerando movimentos incorretos postura inadequadas e a promoção de dores. (ALTER ,1999)

$\mathrm{Na}$ avaliação da dor utilizamos o diagrama das áreas dolorosas, ele divide o corpo em 21 regiões que vão desde o pescoço até os tornozelos e pés. O avaliado identificou em cada região se há dor e qual a sua intensidade que pode variar entre nenhum desconforto até a dor intolerável.

Observamos que $60 \%$ dos entrevistados apresentam sensação de dor no corpo que vão desde a algum desconforto até uma dor intolerável, este dado estar presente em muitos estudos no Brasil quando se procura investigar a presença de dor muscular em docentes associando as características desta atividade. (ARAÚJO et. al.,2005).

Verificamos então um baixo nível de flexibilidade e um alto índice de dor muscular nos docentes avaliados, utilizando o Coeficiente de Correlação de Pearson, constatou-se não haver correlação significativa quando associamos a dor ao nível de flexibilidade observamos um $r=-0,38$, ou seja, existe uma relação inversa, então não podemos afirmar que estas dores apresentadas pelos entrevistados estão associadas aos baixos níveis de flexibilidade, conforme (Gráfico 1). 
Gráfico 1. Dor associada a Flexibilidade

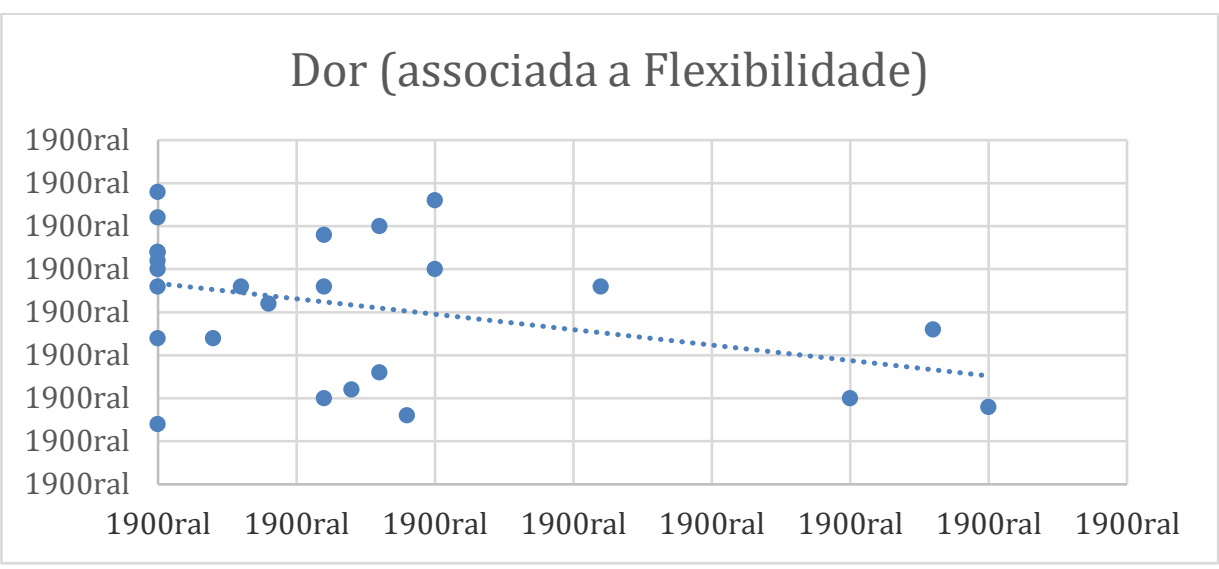

Fonte: PEREIRA, 2021.

Procuramos associar a dor também com a idade de acordo com o Gráfico 2, considerando que o longo tempo de serviço em condições inadequadas pode contribuir com o aparecimento de dores, observamos um $\mathrm{r}=0,48$, ou seja, também não há uma correlação significativa entre a idade com a Dor, mesmo as pessoas mais velhas em tese estarem mais propensas ao aparecimento de dores, profissionais mais jovens já reclamam destes incômodos.

Gráfico 2 Dor associada a Idade

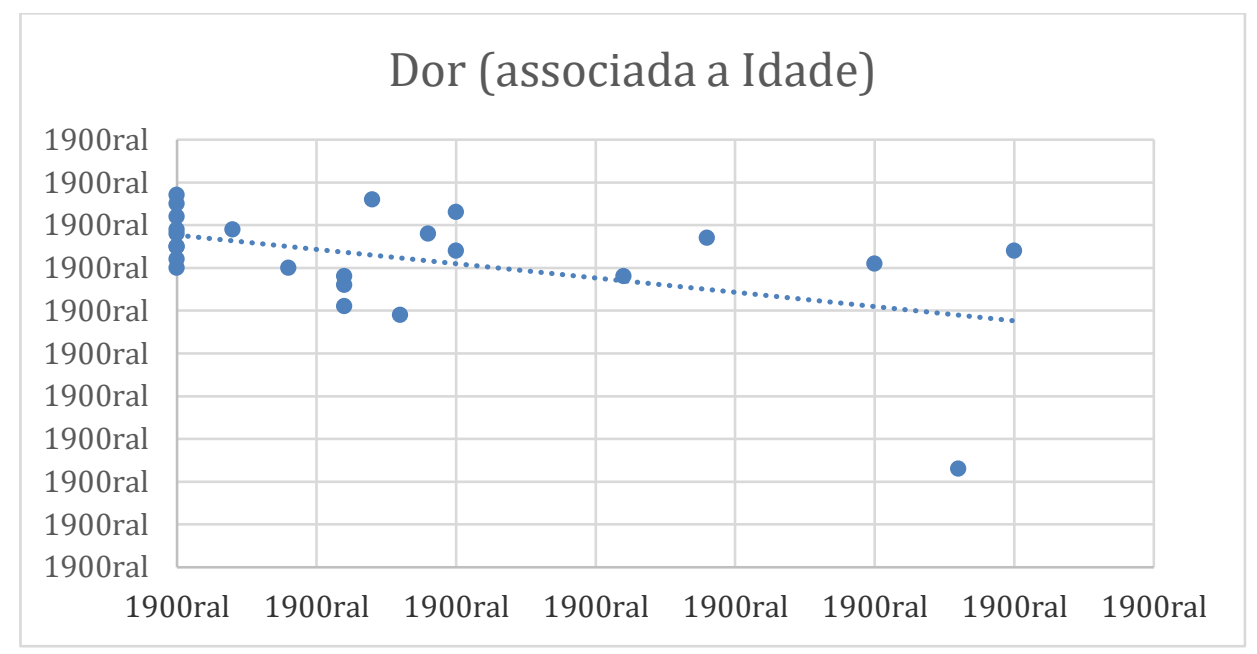

Fonte: PEREIRA, 2021.

A dor muscular é muito presente nos professores, no nosso estudo $40 \%$ dos entrevistados estão entre os níveis 1 a 3, ou seja, nenhum desconforto ao desconforto moderado e $60 \%$ dos entrevistados entre os níveis 4 e 5 , que significa bastante desconforto ao desconforto 
intolerável, estes dados são encontrados em outros estudos. (CARVALHO et al.,2009; CEBALLOS; SANTOS ,2015).

\section{DISCUSSÃO}

Em nossa pesquisa podemos observar que o perfil dos professores da rede Estadual da Mesorregião Metropolitana de Salvador não foge ao perfil dos professores brasileiros, constituído em sua maioria por mulheres, quanto a idade a maioria estar entre 39 e 45 anos, sobre o tempo de serviço notamos que a maioria tem mais de 15 anos de docência, dados também encontrados no estudo feito pelas organizações das nações unidas para a educação, ciência e cultura (UNESCO, 2004).

A atividade docente geralmente nas escolas públicas municipais ou estaduais as aulas acontecem com o professor tendo basicamente como recurso didático o quadro, giz e em algumas disciplinas livros didáticos, essas condições matérias terminam tendo um impacto negativo na atividade docente (SANTOS et al., 2012).

A elaboração de uma aula envolve planejamento, pesquisa e exposição dos conteúdos da disciplina, neste caso os recursos didáticos acabam ajudando nestas tarefas, mas infelizmente estes não estão disponíveis em boa parte das nossas escolas, além destas questões estruturais e pedagógicas os professores assumem diversas demandas na escola acumulando mais sua carga de trabalho (OLIVEIRA, 2004)

Recursos como: data show, lousa magnética e internet instrumentos que facilitariam o trabalho docente e consequentemente menor sobrecarga musculoesquelética não estavam presentes na escola pesquisada, as aulas acontecem com os professores tendo que registrar os conteúdos no quadro e com muitas horas de explanação verbal para que os alunos possam compreender os assuntos.

$\mathrm{Na}$ análise da flexibilidade utilizamos o teste do sentar e alcançar e notamos que mais da metade dos entrevistados apresentam com baixo nível de amplitude articular, apresentaram dificuldades na execução do teste, ter uma boa amplitude de movimento funcional em todas as articulações do sistema musculoesquelético garante uma boa qualidade dos movimentos corporais. (FLECK; KRAMER ,2008).

Quando associamos o desempenho no teste de flexibilidade dos avaliados com a dor não encontramos uma relação direta, também não encontramos correlação entre a dor e a 
idade, verificamos em outros estudos que utilizou-se o teste do banco de Wells para investigar a dor lombar e o nível de flexibilidade não se conseguiu encontrar boa relação, (RIBEIRO ,2006).

O baixo nível de flexibilidade é muito comum em indivíduos sedentários ou que exercem atividades com pouco movimento, o trabalho docente geralmente é feito em condições desfavoráveis levando a um somatório de fatores que podem gerar a dor principalmente quando consideramos a vida do professor fora da escola, como: sedentarismo, jornada dupla, baixos salários, etc.

Trabalhar muito tempo em pé ou sentado, sedentarismo, promover esforços repetitivos em movimentos unilaterais dos membros superiores como nas atividades de escrita, estes comportamentos podem levar ao comprometimento da boa mecânica articular podendo levar posições inadequadas quando somados a movimentos repetitivos (MENDONÇA; ASSUNÇÃO, 2005).

Considerando a atividade docente a região superior do corpo é a mais sobrecarregada não só pela natureza do trabalho do professor, mas geralmente pela alta concentração de gordura na região do abdome o que pode comprometer a coluna vertebral, estas características presentes em muitos docentes podem levar a alta prevalência de dor principalmente na musculatura do troco e região cervical (BRANCO et al., 2012; BARROS et al., 2007).

A dor no dorso e na lombar são percebidas quando os indivíduos ficam por longos períodos em pé, nessa situação ocorre mais atividades nos eretores da coluna, o que gera fadiga muscular e possíveis dores; outra consequência da fadiga nos músculos posturais é a sobrecarga dos ligamentos o que aumenta o estresse sobre os discos vertebrais devido à má postura. (MAEHLER, 2013).

A dor muscular parece ser de causa multifatorial como observamos em alguns que estudos buscam investigar os fatores associados a dor muscular nos docentes, constatamos que esse estado termina comprometendo a qualidade de vida dos professores e suas funções profissionais. (FERNANDES et al. ,2009)

A dor termina gerando limitações na vida dos professores desencadeando outros problemas de saúde e de comportamento, aumenta o nível de estresse, insatisfação 
profissional e redução da capacidade funcional destes indivíduos. (CALIXTO et al.,2015).

Em nossa pesquisa constamos a presença de dor nos docentes mesmo não podendo associa-la com os níveis de flexibilidade, notamos que este é um problema que aflige esta classe de trabalhadores, dado este que pode ser encontrado em boa parte da literatura que discuti este tema, à docência por ser uma profissão cujo desgaste intelectual, emocional e físico está presente, termina expondo esses trabalhadores a riscos de saúde com perdas de suas qualidades de vida em geral. (PAULA; CORIM, 2020).

\section{CONCLUSÃO}

O presente estudo não identificou uma relação entre a dor registrada pelos docentes com o seu nível de flexibilidade, no entanto observamos que essa patologia também estar presente nos professores participantes da pesquisa, assim como em diversos outros estudos sobre este tema, o que reflete uma triste realidade quando investigamos dados sobre a saúde dos docentes no Brasil, deixamos claro a necessidade de mais estudos sobre este problema para que possam servir de alerta para a criação de propostas de intervenção para melhoria da qualidade de vida dos trabalhadores da educação.

\section{REFERÊNCIAS}

1. ALTER, Michael J. Ciência da flexibilidade. $2^{\mathrm{a}}$ ed. Porto Alegre: Artmed, 1999.

2. ARAÚJO T. M; SENA I. P; ARAÚJO E. M. Mal-estar docente: avaliação de condições de trabalho e saúde em uma instituição de ensino superior. Revista Baiana Saúde Pública, Salvador, v.29, n.1, p. 6-21, jan/jun. 2005

3. BARROS, M. E; DOROTÉIA, C. Z; FERNANDA, S.A; ROBERTA Z. I; VIVIAN G.V.A. Saúde e Trabalho docentes. a escola como produtora de novas formas de vida. Trabalho, Educação e Saúde, Rio de Janeiro, v.5, n.1, p.105123, jun. 2007

4. BORSOI, I. C. F; Trabalho e produtivismo: saúde e modo de vida de docentes de instituições públicas de Ensino Superior. Caderno Psicologia Sociologia do Trabalho, São Paulo, v.15, n.1, p. 81-100, jun. 2012

5. BRANCO J. C; SILVA, F.G; JANSEN, S.K; GIUSTI, P.H. Prevalência de sintomas osteomusculares em professores de escolas públicas e privadas do ensino fundamental. Fisioterapia em Movimento, Curitiba, v. 24, n. 2, p. $307-$ 314, jun. 2011

6. BRASIL. Ministério da Saúde. Secretaria de Vigilância em Saúde. Departamento de Vigilância em Saúde Ambiental e Saúde do Trabalhador. Dor relacionada ao trabalho: Lesões por esforços repetitivos (LER) Distúrbios osteomusculares relacionados ao trabalho (Dort). Brasil, 2012. Disponívelem:https://bvsms.saude.gov.br/bvs/publicacoes/dor_relacionada_\%2 Otrabalho_lesoes_ler.pdf . Acessado em: 08 de julho de 2021 
7. BULL FC, et. al., World Health Organization 2020 guidelines on physical activity and sedentary behaviour. British Journal of Sports Medicine, Genova, v. 54, p. 1451-1462, dez 2020

8. CALIXTO, M. F; GARCIA, P. A; RODRIGUES, D. S;ALMEIDA, P.H.T.Q. Prevalência de sintomas osteomusculares e suas relações com o desempenho ocupacional entre professores do ensino médio público. Caderno de Terapia Ocupacional UFSCar, São Carlos, v.23, n. 3, p. 533-542, 2015

9. CARVALHO, M. R. V. Perfil do professor da educação básica. Instituto Nacional de Estudo e Pesquisas Educacionais Anísio Teixeira, Brasília, INEP/MEC, 2018.

10. CEBAllos, A. G. C; SANTOS G. B. Fatores associados à dor musculoesquelética em professores: Aspectos sociodemográficos, saúde geral e bem-estar no trabalho. Revista Brasileira de Epidemiologia, São Paulo, v. 18 n.3, p.702-715, jul/set. 2015

11. CORLETT, E.N.; MANENICA, I. The effects and measurement of working postures. Applied Ergonomics, v. 11, n. 1, p.7-16, mar. 1980

12. COSTA, M. S.G. A; BARBOSA, N.D; CARRARO, P. R. A importância do trabalho do psicólogo escolar aos docentes em escolas públicas. Revista Eixo, Brasília, v. 3, n. 2, p.73-80, jul/dez. 2014

13. FERNANDES, M. H; ROCHA, V. M; OLIVEIRA, A.G.R.C. Fatores associados ä prevalência de sintomas osteomusculares em professores. Revista de Salud Pública, Colômbia, v. 11, n. 2, p. 256-267, mar, 2009

14. FLECK Steven J; KRAEMER William J. Fundamentos do Treinamento de Força Muscular. 3nd ed. Porto Alegre: Artmed,2008.

15. GIL Antônio C. Como elaborar projetos de pesquisa. São Paulo: Atlas, 2008.

16. GUEDES Dartagnan P.; GUEDES Joana E.P. Exercício Físico na promoção da saúde. Londrina: Midiograf,1995.

17. HOWLEY Eward T.; FRANKS B. D. Manual de Condicionamento Físico. 5nd ed. Porto Alegre: Artmed, 2008.

18. IIDA Itiro. Ergonomia Projeto e Produção. 2nd ed. São Paulo: Editora Edgar Blücher, 2005.

19. JEFFERSON PC. Et al. Prevalência de dor musculoesquelética em professores. Revista brasileira de epidemiologia.2009; 12(4):604-614.

20. MAEHLER Paula. Estudo das sobrecargas posturais em acadêmicos de odontologia da Universidade Estadual do Oeste do Paraná-Unioste-Cascavel (Monografia Curso de Odontologia). Universidade Estadual do Oeste do Paraná, Cascavel, 2003.

21. MENDONÇA, J. H. P.; ASSUNÇÃO, A. A. Associação entre distúrbios do ombro e trabalho: breve revisão da literatura. Revista Brasileira de Epidemiologia, São Paulo, v. 8 n. 2, p.167-176. Jun, 2005

22. MONTEIRO, Walace D.; FARINATTI, Paulo T. Fisiologia e Avaliação funcional. Rio de Janeiro: Sprint, 1999.

23. OLIVEIRA, D. A. A reestruturação do trabalho docente: precarização e flexibilização. Educação Sociedade, Campinas, v. 25, n. 89, p. 1127-1144, set/dez, 2004

24. PAULA, G. M; CORIM T. P. A dor lombar como indicador de alteração na qualidade de vida no trabalho de docentes universitários: uma revisão da literatura. Brazilian Journal of Development, Curitiba, v. 6, n. 10, p. 7490574921, out. 2020 
25. PEREIRA, A.L.P. Relações entre estilo de vida e qualidade de vida no trabalho: um estudo com os profissionais técnicos administrativos do Instituto Federal Baiano - Campus Santa Inês. Brazilian Journal of Development, Curitiba, v. 6, n. 11, p. 8703487048, nov. 2020

26. POLACHINI, L. O; FUSAZAKI, L; TELLINI, G.G; TAMASO, M; MASIERO, D. Estudo comparativo entre três métodos de avaliação do encurtamento de musculatura posterior de coxa. Revista Brasileira de Fisioterapia. São Carlos, v.9, n. 2, p.187-93, mai/ago. 2005

27. RIBEIRO, W. G. A relação entre lombalgia e o teste de sentar e alcançar. Revista eletrônica da Escola de Educação Física e Desportos - UFRJ, Rio de Janeiro, v. 2, n. 2,p. $102-114$, jul/dez. 2006

28. SANTOS, R. M; NASCIMENTO, M. A; MENEZES, J.A. Os sentidos da escola pública para jovens pobres da cidade do Recife. Revista Latinoamericana de Ciencias Sociales, Niñez y Juventud, Manizales, v. 10, n. 1, p. 289 -300, jan/jun, 2012

29. STANDAERT, C.J; FRIEDLY, J; ERWIN, M. W; LEE, M.J; RECHTINE, G; HENRIKSON N. B; NORVELL, D.C. Comparative effectiveness of exercises, acupunture, and spinal manipulation for low back pain. Spine, v. 36, n.21, p.120130. Out, 2011.

30. STEVEN, J. F; WILliAM, J. K. Fundamentos do Treinamento de Força. 3nd ed. Porto Alegre: Artmed, 2006.

31. SOUZA,D. G ; MIRANDA, J.C ; GONZAGA, G.R ; SOUZA, F.C. Desafios da prática docente. Revista Educação Pública, Rio de Janeiro, v.17, n.19, p.1-4, out, 2017

32. TACON, K. C. B; COSTA, W.S; VENTO, D.A; VILAR, W. D. B; FERNANDES, V. L. S; BARROS, T. C; OLIVEIRA, L. N. Avaliação da dor lombar correlacionada ao encurtamento dos isquiotibiais em docentes de uma instituição de Ensino Superior. Revista da Sociedade Brasileira de Clinicas Medicas, Goiânia, v. 15, n.1, p.21-26, jan/mar, 2017

33. UNESCO. 2004. O perfil dos professores brasileiros: o que fazem, o que pensam, o que almejam... Disponível em: http://me.precog.com.br/bctexto/obras/ue000027.pdf Acessado em: 24 agosto 2021.

34. WELLS, K.F; DILLON, E. K. The Sit and Reach-A Test of Back and Leg Flexibility. Research Quarterly. American Association for Health, Physical Education and Recreation, Washington, v. 23, n.1, p. 115-118, 1952 